\title{
Revisiting the red supergiant progenitors of core-collapse supernovae
}

\author{
Schuyler D. Van Dyk \\ Caltech, 100-22, Pasadena, CA 91125 USA \\ email: vandyk@ipac.caltech.edu
}

\begin{abstract}
I will present a reanalysis of the properties of the red supergiant (RSG) progenitors of several extragalactic core-collapse, Type II-Plateau supernovae, including SN 2012aw, SN 2013ej, SN 2004et, and SN 2008bk. The observed spectral energy distributions (SEDs) for these stars, from the optical to the infrared, are compared with model SEDs for Galactic RSGs, as well as with the observed SEDs of analogous RSGs in Local Group galaxies. As a result, new estimates of the stellar temperatures and luminosities for the progenitors are obtained, and through comparison with recent massive stellar evolutionary tracks, new inferences are made for the progenitors initial masses. I will place these in the context of the RSG SN progenitor mass spectrum and of the so-called "red supergiant problem."
\end{abstract}

Keywords. supernovae: individual (SN 2008bk, SN 2012aw, SN 2013ej); stars: evolution; stars: luminosity function, mass function; galaxies: individual (NGC 628, NGC 3351, NGC 7793); galaxies: stellar content

\section{Summary}

Van Dyk et al. (2012a) found that the low-luminosity SN II-P 2008bk experienced low reddening and that its cool $\left(T_{\text {eff }} \approx 3600 \mathrm{~K}\right)$ RSG progenitor had an initial mass $M_{\text {ini }} \approx 8-8.5 M_{\odot}$. Subsequently, Maund et al. (2014) concluded that the SN progenitor was substantially more reddened, and therefore was hotter $\left(T_{\text {eff }} \sim 4330 \mathrm{~K}\right.$; more in line with the RSG temperature scale by Davies et al. 2013), more luminous, and more massive $\left(M_{\text {ini }} \approx 12.9 M_{\odot}\right)$. Our recent analysis, correcting for the brightness of stars around the SN (from Van Dyk 2013), continues to indicate that the RSG was cooler $(\sim 3500 \mathrm{~K})$ and had $M_{\text {ini }} \approx 8-9 M_{\odot}$. As an update to Van Dyk et al. (2012b) and Fraser et al. (2012), based on DUSTY (Ivezic \& Elitzur 1995) modeling of the RSG dust shell and comparison with M33 RSGs (Drout et al. 2012), we find that the progenitor of the SN II-P 2012aw had $M_{\text {ini }}$ more likely $\sim 13-14 M_{\odot}$. We also find that the progenitor of the SN II-P 2013ej (Fraser et al. 2014), also from DUSTY modeling and a M33 RSGs comparison, was likely more massive, $M_{\text {ini }}>15 M_{\odot}$. The presentation was too brief to discuss SN II-P 2004et.

\section{References}

Davies, B., Kudritzki, R. P., Plez, B., Trager, S., Loncon, A., Gazak, Z., et al. 2013, ApJ, 767, 3 Drout, M. R., Massey, P., \& Meynet, G. ApJ, 750, 97

Fraser, M., Maund, J. R., Smartt, S. J., Botticella, M.-T., et al. 2012, ApJ, 759, L13

Fraser, M., Maund, J. R., Smartt, S. J., Kotak, R., Lawrence, A., et al. 2014, MNRAS, 439, L56

Ivezic, Z. \& Elitzur, M. 1995, ApJ, 445, 415

Maund, J. R., Mattila, S., Ramirez-Ruiz, E., \& Eldridge, J. J. 2014, MNRAS, 438, 1577

Van Dyk, S. D., Davidge, T. J., Elias-Rosa, N., Taubenberger, S., et al. 2012a, AJ, 143, 19

Van Dyk, S. D., Cenko, S. B., Poznanski, D., Arcavi, I., Gal-Yam, A., et al. 2012b, ApJ, 756, 131

Van Dyk, S. D. 2013, AJ, 146, 24 